\title{
Coherent tunneling of mixed state hole wave packets in coupled quantum well structures
}

\author{
Vasu Sankaran and Jasprit Singh \\ Center for High Frequency Microelectronics, Department of Electrical Engineering and Computer Science, \\ University of Michigan, Ann Arbor, Michigan 48109-2122
}

(Received 25 June 1990; accepted for publication 7 January 1991)

\begin{abstract}
The time-dependent Schrödinger equation is solved numerically to study the coherent tunneling of hole wave packets in asymmetric coupled quantum wells. The importance of selection rules and band mixing is evident in the extremely low rates of the wave-packet leakage from heavy-hole state to a resonant light-hole state at zero in-plane wave vector $\left(\mathbf{k}_{\|}\right)$. But these rates increase dramatically away from $\mathbf{k}_{\|}=0$, when the hole states acquire mixed character, and rapidly become comparable to the heavy-hole to heavy-hole resonant tunneling rates. The effect of inhomogeneous level broadening arising from well size fluctuations in multicoupled quantum well systems is shown to greatly reduce the effective tunneling rates near resonance.
\end{abstract}

Tunneling in quantum well structures has recently gained considerable interest. ${ }^{1-4}$ This is especially true for cases where the representation of electronic states involves bases states of differing symmetry, and the character of the electron or hole wave function changes across the heterostructure. This is the case, for instance, when a direct bandgap electron ( $\Gamma$ type) escapes through an indirect bandgap barrier ( $X$ or $L$ type). Similar considerations arise in hole tunneling when the hole state is described using a degenerate $\mathbf{k} \cdot \mathbf{p}$ formalism, as is appropriate in III-V materials like GaAs where the top of the valence band is fourfold degenerate. This letter, focuses on the tunneling of a hole wave packet in an asymmetric coupled quantum well (CQW) shown in Fig. 1(a). Such structures are often chosen by experimentalists because of the ease with which a hole wave packet can be selectively introduced in one well and the coherent tunneling studied with application of an electric field. Figure 1(b) shows the calculated subband energies over a range of electric field strengths in a 56/ $48 / 88 \AA \mathrm{GaAs} / \mathrm{Al}_{0,3} \mathrm{Ga}_{0,7}$ As double quantum well calculated at $\mathbf{k}_{\|}=0$. As seen from this figure, the heavy-hole ( $\mathrm{HH}$ ) state $\mathrm{HH} 1$ in the wide well (WW) can be resonantly tuned to the light-hole ( $\mathrm{LH})$ state LH1 in the narrow well (NW) at $31 \mathrm{kV} / \mathrm{cm}$ and to $\mathrm{HH} 2(\mathrm{NW}$ ) at $52 \mathrm{kV} / \mathrm{cm}$. A wave packet can be injected by pulsed laser near the HH1(WW) state and the luminescence decay can then study the tunneling escape of injected carriers. Some controversy exists at present on whether or not the $\mathrm{HH}$ wave packet can tunnel through the LH state when they are resonantly lined up by the electric field. Leo et al..$^{5}$ have observed essentially no tunneling when the HH state is lined up with the LH state, while Norris ${ }^{6}$ and Liu et al. ${ }^{7}$ report rapid tunneling under these conditions. The tunneling results are obtained from time-resolved photoluminescence experiments and since these techniques measure the decay of carriers in a particular well they depend upon coherent tunneling and scattering processes. Since scattering is somewhat sample dependent, it is important to study the intrinsic process of coherent tunneling alone.

We will briefly describe our approach for studying the time evolution of multiband hole wave packets, which is an extension of a well-known method used to study the prop- agation of electron wave packets whose time evolution is determined by a scalar Schrödinger equation. ${ }^{8}$ We choose to describe the hole states using the multiband effective mass theory of Luttinger and Kohn. ${ }^{9}$ In the envelope function approximation resulting from a four-band description, the time-dependent hole state is given by

$$
\psi\left(\mathbf{k}_{\|}, \mathbf{r}, t\right)=\frac{e^{\mathbf{k}_{\|} \cdot \mathbf{r}_{\|}}}{2 \pi} \sum_{v} f_{v}\left(\mathbf{k}_{\|}, z, t\right) u_{v 0}(\mathbf{r}),
$$

where $u_{v 0}$ are the zone center Bloch functions of the valence band, $f_{v}$ the corresponding envelop functions, $\mathbf{k}_{\|}$the in-plane wave vector, $\mathbf{r}_{\|}$the in-plane coordinate, and $z$ the
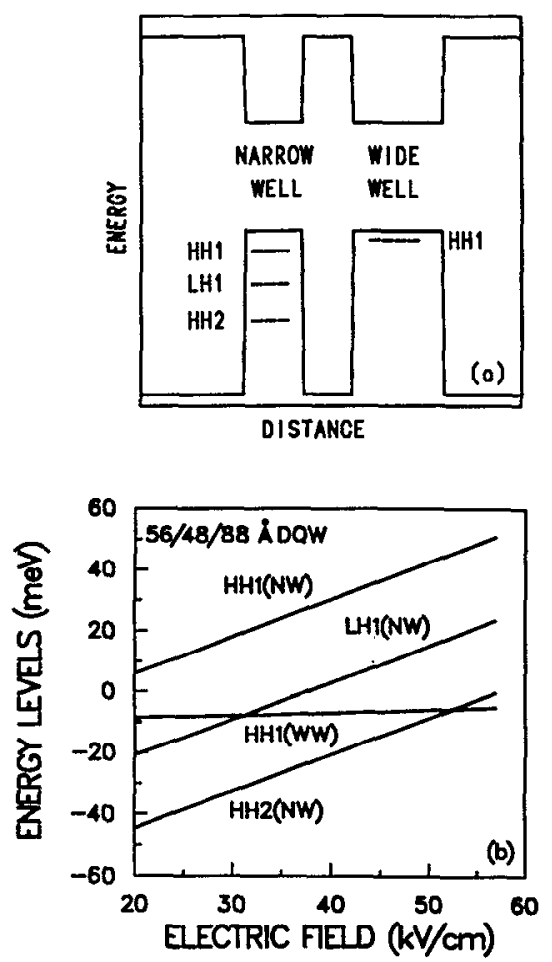

FIG. 1. (a) Schematic band diagram and hole subbands in an asymmetric double quantum well structure, and (b) subband levels as a function of electric field in a $56 / 48 / 88 \AA \mathrm{GaAs} / \mathrm{Al}_{0.3} \mathrm{Ga}_{0.7}$ As double quantum well calculated at $\mathbf{k}_{\|}=0$. Center of the wide well is set to zero energy. 
coordinate in the growth direction. The Bloch functions $u_{20}$ transform as the $|3 / 2, \pm 3 / 2\rangle(\mathrm{HH})$ and $\mid 3 / 2, \pm 1 /$ 2) ( $\mathrm{LH})$ angular momentum states. This is simply labeled by the subscript $v$, with the notation $v=1,2,3$, and 4 for the $(3 / 2,3 / 2),(3 / 2,1 / 2),(3 / 2,-1 / 2)$, and $(3 / 2,-3 / 2)$ components, respectively.

The envelope functions evolve under the multiband effective mass Hamiltonian according to the coupled set of partial differential equations: ${ }^{10}$

$$
\begin{aligned}
i \hbar \frac{\partial}{\partial t} f_{v}\left(\mathbf{k}_{\|}, z, t\right)= & \sum_{v^{*}}\left[H_{v v^{\prime}}\left(\mathbf{k}_{\|},-i \frac{\partial}{\partial z}\right)\right. \\
& \left.+V(z) \delta_{v^{*}}\right] f_{v^{\prime}}\left(\mathbf{k}_{\|}, z, t\right) .
\end{aligned}
$$

Here $V(z)$ is the potential of the top of the valence band across the heterostructure and $H_{y v^{\prime}}$ is the Luttinger kinetic matrix ${ }^{9}$ which acts as a matrix differential operator. Using the assumption that the envelope functions do not vary appreciably over the dimensions of the unit cell, the probability of finding a hole within a region extending from $z_{1}$ to $z_{2}$ is taken to be

$$
P_{h}(t)=\sum_{v} \int_{z_{1}}^{z_{2}} d z\left|f_{v}(z, t)\right|^{2}
$$

In the wave packet method, we construct the initial wave packet $\psi(r, 0)$ as a localized state that can well represent the initial state of the charge carriers in an actual experiment. For a scalar Schrödinger equation, a formal solution to the time evolution of a wave packet in a timeindependent potential is given by ${ }^{11}$

$$
\psi(\mathbf{r}, t)=e^{-i H t / \hbar} \psi(\mathbf{r}, 0)
$$

A numerically stable procedure for solving this equation by discretizing the time interval of interest into infinitesimal steps $\delta t$, and using a unitary, second-order accurate approximation to the evolution operator is discussed in Ref. 8. One obtains the implicit equation

$$
(1+i H \delta t / 2 \hbar) \psi(\mathbf{r}, t+\delta t)=(1-i H \delta t / 2 \hbar) \psi(\mathbf{r}, t)
$$

for the evolution of the wave function. We have extended this procedure to our multiband representation of the hole states. The time evolution of the envelope functions is determined by successively solving the set of equations

$$
\begin{array}{r}
\left(1+\frac{i[H] \delta t}{2 \hbar}\right)\left[\begin{array}{c}
f_{3 / 2,3 / 2}(z, t+\delta t) \\
f_{3 / 2,1 / 2}(z, t+\delta t) \\
f_{3 / 2,-1 / 2}(z, t+\delta t) \\
f_{3 / 2,-3 / 2}(z, t+\delta t)
\end{array}\right] \\
=\left(1-\frac{i[H] \delta t}{2 \hbar}\right)\left[\begin{array}{c}
f_{3 / 2,3 / 2}(z, t) \\
f_{3 / 2,1 / 2}(z, t) \\
f_{3 / 2,-1 / 2}(z, t) \\
f_{3 / 2,-3 / 2}(z, t)
\end{array}\right],
\end{array}
$$

where $[H]$ is now the $4 \times 4$ Luttinger Hamiltonian. This set of equations is solved by the finite difference method at each time step.
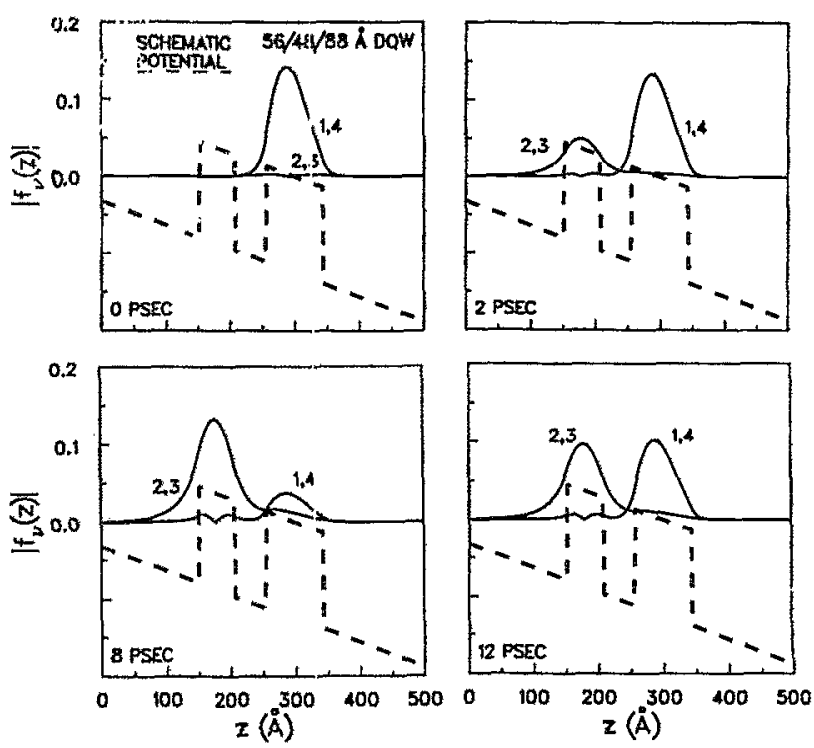

FIG. 2. Time evolution of envelope functions of a HH1(WW) wave packet in the $56 / 48 / 88 \AA$ double quantum well structure under an applied field of $31 \mathrm{kV} / \mathrm{cm}$ when LHI(NW) and HHI(WW) are in resonance. Results are for $k_{x}=0,002 \AA^{-1}, k_{y}=0$.

For the purposes of this letter, we have not included any incoherent processes into account and it is to be noted that for this ideal case the wave packet essentially oscillates between the two coupled wells. However, we assume the presence of relaxation mechanisms by which the escaping holes can deexcite to lower energy levels in the NW, preventing them from tuinneling back. Assuming the tunneling escape of carriers to be described by an exponential decay law, tunneling times $\tau$ are obtained from the initial decay rate of probability in the $W W$ :

$$
\tau=\frac{P(0)}{(P(0)-P(t))} t_{*}
$$

where $P(t)$ is the total probability in the WW at time $t$. Thus, $\tau$ may be taken to be just a measure of the initial decay rate of probability in the WW. An example of the wave-packet oscillation is shown in Fig. 2 that depicts the time evolution of a wave packet characterized by $k_{x}=0.002 \AA^{-1}, k_{y}:=0$, under an applied electric field such that HH1(WW) and LH1(NW) states are in resonance. The time development of the initially small LH component can be seen from these results.

Figure 3(a) depicts the tunneling time of a hole wave packet initially created with $\mathbf{k}_{\|}=0$ near the HH1(WW) state as a function of the electric field. The tunneling time abruptly decreases only when the HH1(WW) state lines up with the $\mathrm{HH} 2$ (NW) state at $52 \mathrm{kV} / \mathrm{cm}$. At $\mathbf{k}_{\|}=0$ there is essentially no tunneling when the $\mathrm{HH} 1$ (WW) state lines up with the LH1 (NW') state. But as we move away from $\mathbf{k}_{\|}=0$ the Luttinger Hamiltonian introduces a coupling between $\mathrm{HH}$ and $\mathrm{LH}$ components of the hole wave function, causing the tunneling rates to increase rapidly as shown in Fig. 3(b) in the appearance of a second resonance when the $\mathrm{HH}$ l(WW) state lines up with 

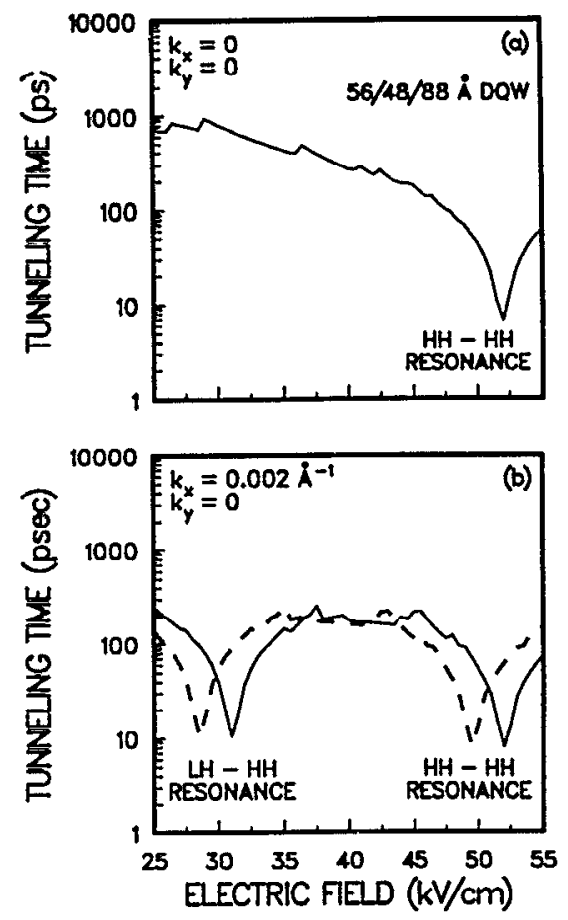

FIG. 3. Tunneling time as a function of electric field for the $56 / 48 / 88 \AA$ double quantum well structure (a) with $\mathrm{k}_{\|}=0$, and (b) with $k_{x}=0.002$ $\AA-1, k_{y}=0$ (solid line). The dotted line in (b) is the corresponding result when the narrow well width is increased by a monolayer.

LH1 (NW). Note that the value of $\mathbf{k}_{\|}$used for the results of this figure $\left(k_{x}=0.002 \AA^{-1}, k_{y}=0\right)$ yields an initial state whose energy is only $0.1 \mathrm{meV}$ above the energy of the corresponding state at $\mathbf{k}_{\|}=0$. Such states may be occupied under most experimental situations.

Finally, Fig. 4 is a schematic 3D plot of the tunneling times computed over a wide range of electric field and transverse wave vector. It is clear from these figures that at finite $\mathbf{k}_{\|}$values the $\mathrm{HH} 1$ (WW) state tunnels quite rapidly

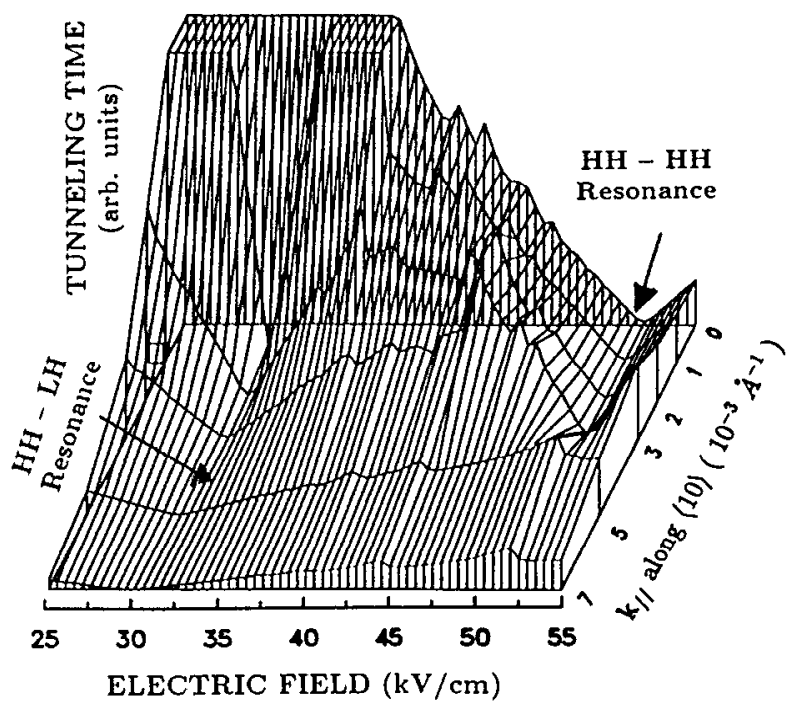

FIG. 4. Schematic plot of tunneling time as a function of electric field and in-plane wave vector for the $56 / 48 / 88 \AA$ double quantum well. to the LH1(NW) state. We stress that these tunneling times pertain directly to the process of coherent tunneling as opposed to assisted, incoherent processes, and arise from the off-diagonal coupling terms of the Luttinger Hamiltonian, which are nonzero for finite $\mathbf{k}_{\|}$. In an actual experiment, the coherent tunneling discussed above between the ground state in one well and an excited state in the adjacent well will be observed as a decay of hole population in the starting well only if there is a relaxation mechanism by which the escaping holes can deexcite to lower energy levels in the target well, preventing them from tunneling back to the starting well. For high-quality samples with low impurity concentration and abrupt interfaces, the dominant low-temperature scattering mechanism is expected to be optical phonon emission for relaxation from the excited LH1(NW) state to the ground HH1(NW) state. However, if the energy separation between LH1(NW) and $\mathrm{HH} 1(\mathrm{NW})$ is less than the polar optical phonon energy, the relaxation may depend on less difficult mechanisms, in which case the wave packet simply oscillates between the two wells, and the observed decay times will be much longer than expected from the period of these coherent oscillations.

We also note that experiments on such coupled wells use a series of such wells to increase the photoluminescence signal. If these wells have some structural fluctuations, the resonance will not be as sharp. The dotted curve in Fig. 3 (b) shows what the tunneling rates are if the narrow well size increases in width by a monolayer $(\approx 3 \AA)$. It is quite clear that such a small fluctuation can change the effective tunneling rate by an order of magnitude with rapid tunneling in one coupled well and very slow tunneling in another one with slightly different parameters.

In summary we have shown that the tunneling between HH and LH states is suppressed only for zero in-plane wave vector. At finite values of $\mathbf{k}_{\|}$, the initial state is close in energy to the $\mathbf{k}_{\|}=0$ case, but tunneling is much more rapid and decay times are very similar for HH-LH and HH-HH resonances.

This work was supported by the U. S. Army Research Office (grant No. DAAL03-87-K-0007). Part of this work was also supported by the Air Force URI program.

${ }^{1}$ T. B. Norris, N. Vodjdani, B. Vinter, C. Weisbuch, and G. A. Mouron, Phys. Rev. B 40, 1392 (1985).

${ }^{2}$ S. Luryi, Solid State Commun. 65, 787 (1988).

${ }^{3}$ D. Y. Oberli, J. Shah, T. C. Damen, C. W. Tu, T. Y. Chang, D. A. B. Miller, J. E. Henry, R. F. Kopf, N. Sauer, and A. E. DiGiovanni, Phys. Rev. B 40, 3028 (1989).

${ }^{4}$ M. Nido, M. G. W. Alexander, W. W. Ruhle, T. Schweizer, and K. Kohler, Appl. Phys. Lett. 56, 355 (1990).

${ }^{5}$ K. Leo, J. Shah, J. P. Gordon, T. C. Damen, D. A. B. Miller, C. W. Tu, J. E. Cunningham, and J. E. Henry (unpublished).

${ }^{6}$ T. B. Norris, Ph. D. thesis, University of Rochester, 1989.

${ }^{7}$ H. W. Liu, R. Ferreira, G. Bastard, C. Delalande, J. F. Palmier, and B. Etienne, Appl. Phys. Lett. 54, 2082 (1989).

${ }^{8}$ A. Goldberg, H. M. Schey, and J. L. Schwartz, Am. J. Phys. 35, 177 (1967).

${ }^{9}$ J. M. Luttinger and W. Kohn, Phys. Rev. 97, 869 (1955).

${ }^{10} \mathrm{~S}$. Datta, Quantum Phenomena, Modula Series on Solid State Devices, Vol. VIII (Addison Wesley, New York, 1989).

${ }^{1}$ L. I. Schiff, Quantum Mechanics, 3rd ed. (McGraw-Hill, New York, 1949). 